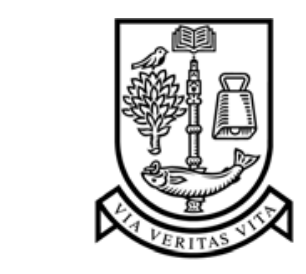

UNIVERSITY

of

GLASGOW

Barkhuus, M. and Chalmers, M. and Hall, M. and Tennent, P. and Bell, M. and Sherwood, S. and Brown, B. (2005) Picking pockets on the lawn: the development of tactics and strategies in a mobile game. Lecture Notes in Computer Science 3660:pp. 358-374.

http://eprints.gla.ac.uk/3424/ 


\title{
Picking Pockets on the Lawn: The Development of Tactics and Strategies in a Mobile Game
}

\author{
Louise Barkhuus, Matthew Chalmers, Paul Tennent, Malcolm Hall, Marek Bell, \\ Scott Sherwood and Barry Brown \\ Department of Computing Science, University of Glasgow, \\ University Avenue, Glasgow, G12 8QQ, UK \\ matthew@dcs.gla.ac.uk
}

\begin{abstract}
This paper presents Treasure, an outdoor mobile multiplayer game inspired by Weiser's notion of seams, gaps and breaks in different media. Playing Treasure involves movement in and out of a wi-fi network, using PDAs to pick up virtual 'coins' that may be scattered outside network coverage. Coins have to be uploaded to a server to gain game points, and players can collaborate with teammates to double the points given for an upload. Players can also steal coins from opponents. As they move around, players' PDAs sample network signal strength and update coverage maps. Reporting on a study of players taking part in multiple games, we discuss how their tactics and strategies developed as their experience grew with successive games. We suggest that meaningful play arises in just this way, and that repeated play is vital when evaluating such games.
\end{abstract}

\section{Introduction}

The study and design of games has added diversity to many areas of ubicomp research. Games are not only a subject worthy of academic attention in themselves [11], they introduce challenges in terms of designing enjoyable experiences. They also lead to technical challenges in implementing distributed ubicomp systems, and players' engagement can lead to new patterns of use that reveal system strengths and weaknesses. For example, Uncle Roy All Around You [2] delivered generalisable results concerning positioning systems and the use of self-reporting, and Real Tournament [10], a simple 'shooter' game, explored IPv6 and issues such as host mobility, security, content delivery and wireless overlay networks.

In this paper, we present our experiences with Treasure, a mobile multiplayer game. Treasure involves both competition and collaboration among players using PDAs with GPS and 802.11 wireless networks. While existing ubicomp games have almost exclusively been trialled in single games, we explored the changing use of game features as players learned about the game through multiple plays. We focus on how their experience of multiple games changed their play and their understanding of the game as well as how the play gives rise to more complex forms of co-operation and competition. 
We start the paper by describing the game's motivation, before outlining the technical and design features of the system. We then present our results from the user trials, describing how the tactics and strategies of players changed as game play developed. We then reflect on these trials, and how repeated trials can be used to inform designers of good game experiences. Lastly we outline our ongoing work that builds on Treasure to develop more mobile games with lengthy game playing periods.

\section{The Motivation and Design of the Game}

Applications may be built to be uniform and 'seamless', but their infrastructure often shows through in interaction, with features such as errors in positioning systems and the limits of $802.11 \mathrm{WiFi}$ coverage becoming apparent in system use [4, 9]. Although this phenomenon is often considered to be negative, we saw the potential to make positive use of it by making the spatial variation of an 802.11 network a central resource in a system design. This infrastructure is a feature presented in the interface, and is also an implicit feature of many interface operations and game actions.

We drew inspiration from Mark Weiser's notions of seams and seamful design [12]. A seam is a break, gap or 'loss in translation' in a number of tools or media, designed for use together as a uniformly and unproblematically experienced whole. Seams often appear when we use different digital systems together, or use a digital system along with the other older media that make up our everyday environment. For example, many applications for mobile computers may be built as if they could be used along with the features of the environment one travels through, e.g. to display web pages about nearby buildings and people. Such applications often assume constant network connectivity, and yet this is not always the case when mobile systems really are mobile: as one walks away from an access point, such systems often crash or become unusable as the wireless connection weakens and then disappears. In urban areas it is likely that there are variations, gaps and overlaps in networks' coverage. The built environment also makes for variations in the accessibility of GPS positioning, due to occlusion and multipath reflections from buildings. Rather than designing the game to be played within one isolated network in an open area, we explored a design in which such features were an explicit and essential part of the game experience.

Although we began with a great variety of game features, devices and roles, an iterative design process led us to progressively simpler designs that concentrated on a small set of simple interactions between players, and a relatively simple map display on PDAs. Earlier designs had other names, including Seamful Game and Bill. We treated the design process, including the user trials, as exploratory. Designs often changed in response to ongoing findings, which were generally reflections from observational studies of system use. We considered this evaluation approach better suited to our design process than one driven by pre-established formal hypotheses.

Before we outline game play and the underlying system, we note that concepts such as seams and seamful design need not be important for players. Particular seams may be apparent in how players play and discuss a system, but they do not necessarily need or want to analyse these design concepts in abstract terms - just as they do not need to 


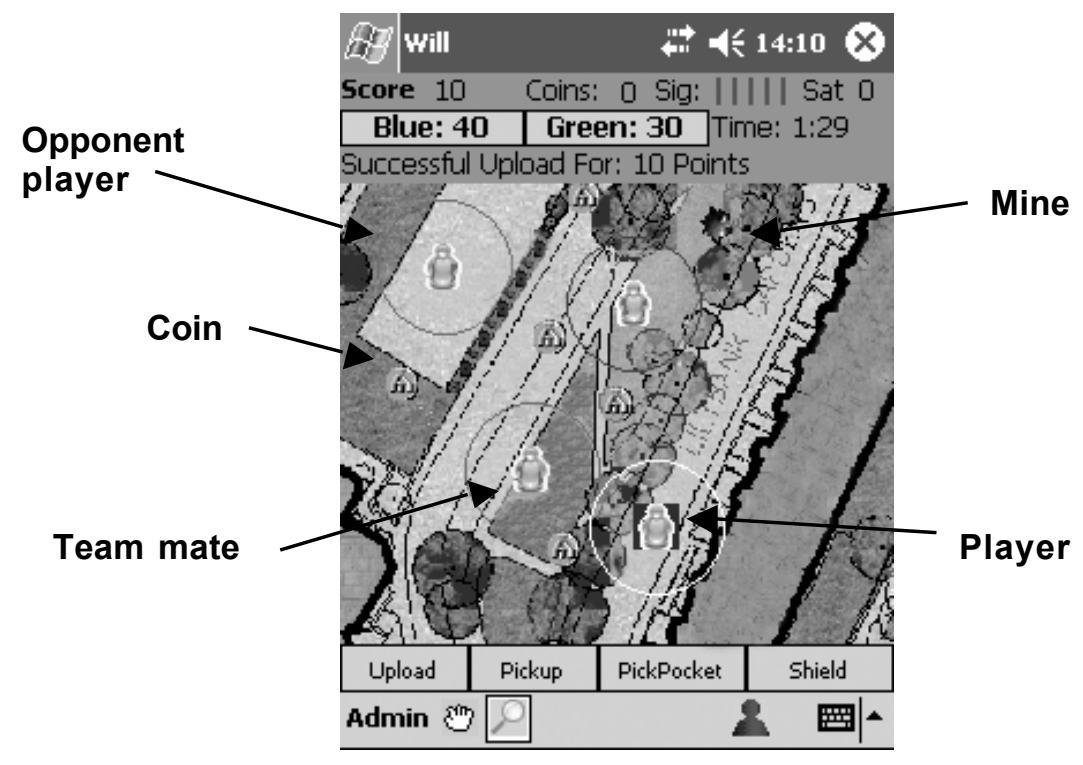

Fig. 1. The game interface. The map shows players' locations, along with coins that are often positioned outside the network. A semi-transparent map layer of green, yellow and red squares builds up as players move around, revealing network coverage, however for printing reasons these have not been included in this figure.

have formal training in the physics of RF propagation or wireless network communication protocols. The way that the game uses or reveals an aspect of ubicomp infrastructure is not meant to be part of a technical education, even though the technology of the game is clearly an element of its design. We aimed for a game that was fun to play, where play could be observed or recorded in ways that would let us, as researchers, better understand the relationship between game play and the system's design.

\subsection{Game Play}

The aim of the game is to collect coins scattered over an urban area and then get them in to the treasure chest (technically, a server). Two teams of two players compete against each other. A clock counts down, and the team with the most coins in their treasure chest at the end wins the game. The coins can only be seen on the map on the players' PDAs (see Fig. 1 for screenshot). Each player's location, the locations of coins and the locations of other players are displayed on his or her map. To pick a coin up, a player has to run over to it and then click the Pickup button. Coins are dropped by the server in all parts of the arena, and a player can pick up coins at any time, but a player can only upload coins (with the Upload button) when he or she is within the wireless network. The chances of a successful coin upload increase with the strength of the wireless network at that location. To succeed in a game, the player must therefore learn which areas are covered by wireless network and which are not. 
The signal strength of the wireless network is shown in the upper right corner of the screen as small bars; when there are few bars and they are red, the signal is low; when there are many bars and they are green, it is high. By moving in and out of areas of network coverage, players also survey the wireless network they are playing with, building up a collective map of signal strength. A high level of signal strength leaves a green trail, a moderate level a yellow trail, and low or no signal colours the area red. In between the coins there are mines, shown as black icons on the PDA map. If a player hits or walks over a mine, he or she loses all the coins on the PDA, and the interface is disabled for 20 seconds, displaying a black countdown screen.

Players can upload coins together and gain double points for their coins. They have to press the Upload button at the same time (technically, within three seconds of each other). A player can also pickpocket another player by walking up close and clicking Pickpocket; he or she then gets all the coins that the other player has (but not the coins that have already been uploaded). If the player wants to prevent pickpocketing, he/she can raise a shield by clicking Shield; this lasts one minute but then has to be recharged. Upload, Pickpocket and Shield only work inside the network, but the Pickup button works anywhere.

\subsection{Technical issues}

The trials reported on in this paper involved four HP iPAQ PDAs running Pocket PC, each with a compact flash GPS unit and built-in 802.11. Each runs a C\# game client, developed with the .NET Compact Framework. The client's interface is shown in Fig. 1. The system also involves a wireless network access point connected to a PC running the game server (again written in $\mathrm{C \#}$ ). The client and server code is instrumented, with each process building up a log of system events such as GUI actions, new GPS positions and other changes to game state. At the end of the game, when the PDA is on the network, the clients' logs are automatically sent to our server.

At any point during a game, a PDA's network connection can be roughly categorised as disconnected, strongly connected or weakly connected. The last of these is the prevalent state; play often takes place on the edge of the network. As a result, we developed a simple messaging subsystem using the UDP protocol rather than the more common TCP. The server 'heartbeats' the game state, broadcasting all game state information (scores, positions etc.) every second across the network. We have found the messaging system to be robust, but we recognise that it would not scale well to high numbers of clients creating large volumes of state information and net traffic.

Another issue associated with the continuous movement in and out of network by clients is the way that the standard Pocket PC wireless drivers may attempt connections to the nearest network. Also, a GUI window pops up when such a connection happens, with a "New Network Found" notification. Since the movement of players often brings them into range of networks other than our own, we created a custom wireless driver that allowed us to 'lock on' to a particular network SSID and connect only to that. Along with the use of static IP addresses, this allowed data connections 
to be established very quickly when clients returned to our network, and increased the chance of a successful connection in areas of weak coverage.

\section{Related Work}

Many mobile multiplayer games have been developed, and several have been run on a large scale in a commercial setting, e.g. Newt Games' Mogi Mogi (www.mogimogi.com). However, a game related closely to ours was Can You See Me Now? (CYSMN), which linked on-line and street players in a chase game [9]. Street players (runners) moved around the game area covered by a game-specific wireless network, and had their positions tracked by GPS. On-line players used arrow keys to move themselves around a $3 \mathrm{D}$ view of the same streets, with icons showing the locations of runners. Similarly, online players' positions were shown on the mobile computers carried by runners. Runners chased on-line players through the city, making their GPS positions match the on-line players' positions i.e. 'catching' them. In playing CYSMN, the variable accuracy of GPS caused problems for street players when trying to catch players in areas of bad GPS coverage. However, through repeated plays and their social interaction, runners became more skilled at using their knowledge of good and bad GPS areas, luring online players into areas of good GPS where catching them was easier. In this way the runners took advantage a limitation - a seam - of the game infrastructure, but the game was not designed to make explicit use of this. This shows a benefit of trials of ubicomp systems that take a longer time or involve repeated use: they can reveal the development of deeper understanding and appropriation of system features, most particularly through social interaction.

The Pirates! game [8] used RF technology to determine the proximity of players to one another and to static resources. This allowed the game to be played either indoors or outdoors. The game mapped an ocean environment on to the rooms and objects in a conference hall, and players took the role of ship commanders, traveling from island to island, and trading and fighting in order to gain wealth. The underlying RF infrastructure was mapped to specific game events so that when a player came close to a RF beacon representing an island, a game event was triggered. In particular, face-to-face interaction was a key part of the game, encouraging some of the social aspects of gaming that can be lost in some computer game designs. Pirates! was tested at a large conference with 31 users and an average number of players in the game arena of four. An implicit part of the Pirates! game was the range, limits and gaps in RF, but Treasure makes similar infrastructure (802.11) a more explicit part of its design.

Another game that influenced our work was NodeRunner (www.noderunner.com), which made use of the wireless network infrastructure existing in a city. As demonstrated at Ubicomp 2003, each team had a PDA equipped with 802.11 and a camera. Teams of players raced against time, logging as many wireless access points as they could and uploading photographic proof of each find to a central server. While NodeRunner made original use of the existing invisible wireless infrastructure, it made no use of the signal beyond the existence of access points. 
Numerous other mobile games have been developed, but here we have focused on the games most relevant to our work; other games are based on different infrastructures such as mobile phone networks or focus on other areas such as education. An overview of a number of these systems and related design issues can be found in [3].

\section{User Trials}

Through our trials we hoped to understand how people used and reacted to a design which makes an element of ubicomp infrastructure an explicit part of an interaction design, a major characteristic of Treasure. We also aimed to apply a lesson from studies of systems such as CYSMN [9], which showed that social interaction often contributes to and reveals how players change and develop new ways of using a system. This occurs as games progress and more experience is gathered and shared. The trials reported here looked at social interaction and multiple plays, with detailed observation intended to reveal the tactics and strategies players developed in the course of multiple games. This point was especially significant to us, as all of our game's earlier trials had been limited to first-time players.

\subsection{Participant Recruitment and Trial Structure}

The participants were recruited in pairs, and in the end we had nine teams of two. The games were set up so no teams played against each other twice. Nine games were played all together, with four teams playing one game, one team playing two and four teams playing three games. All participants were all compensated equally for their time. The participants were all between the age of 17 and 33 with a mean of 23.5; we recruited a few more females than males, but the teams were mixed as well as same sex. Most were students, from a wide variety of disciplines-including three students of computer science. All participants used computers daily.

We ran the games on a fairly large lawn, its surrounding streets and in part of an adjacent car park. This was a good game arena, in that there was considerable open space, trees to hide behind, and relatively little road traffic. The area was about 7000 square metres (64000 square feet). Roughly half of the area had good wireless network coverage. However, since weather has a significant effect on the strength and reach of 802.11 , coverage varied from game to game; hence it is difficult to illustrate a map of average coverage. Generally the right hand side of the map in Fig. 1 had coverage and the left hand side had little or no network coverage. GPS worked well out on the open area of the lawn, but it was difficult to get accurate readings close to the building, adjacent to the lawn, where the 802.11 access point was situated.

Before first-time participants played, they were given a thorough introduction indoors, in a conference room, together with their opponents. One researcher explained the game using a paper prototype of the PDA display for about ten minutes, and then a second researcher entered with the four PDAs. The participants were then given the PDAs, the researchers demonstrated simple manoeuvres such as panning and zooming 


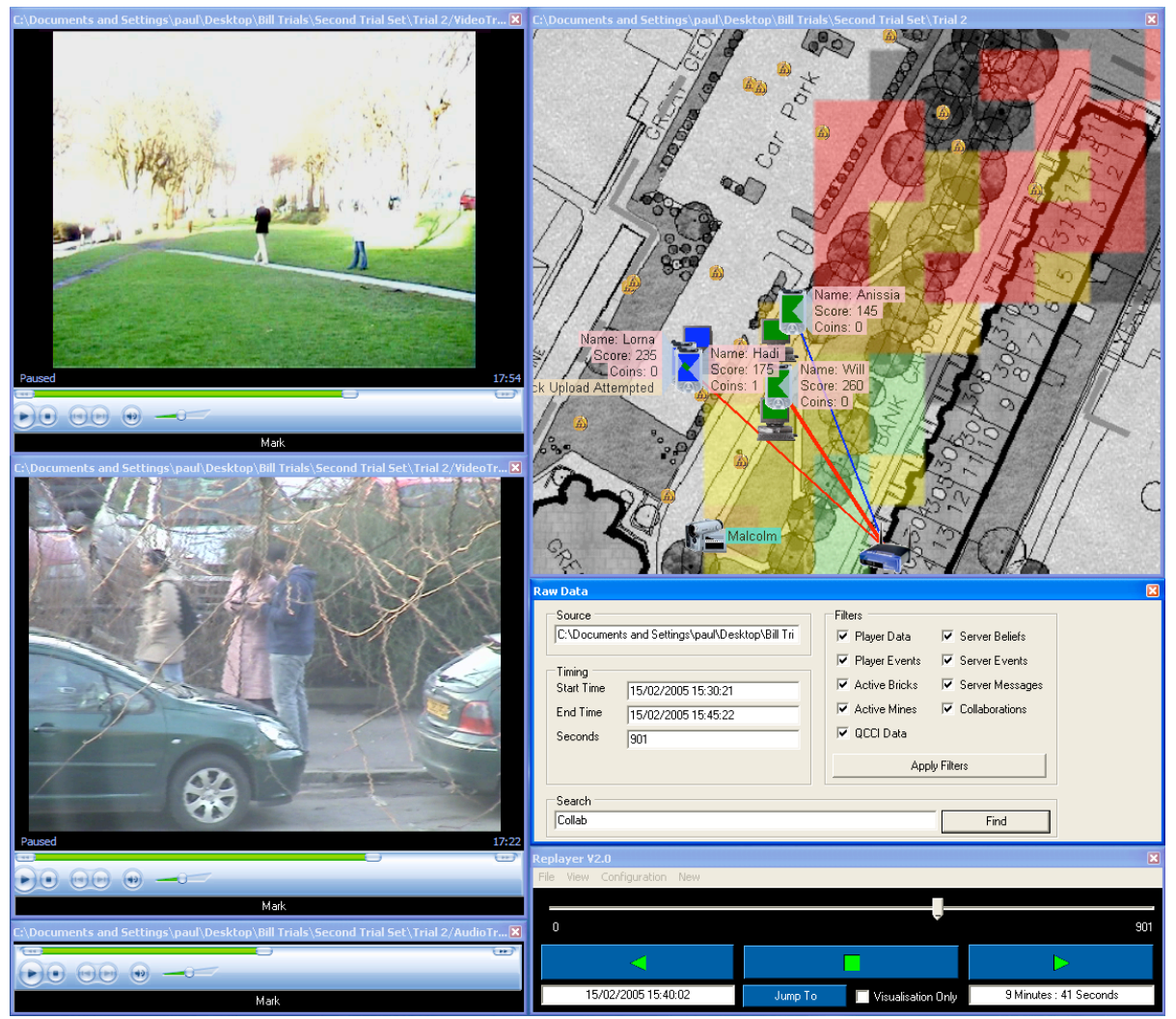

Fig. 2. The Replayer tool is used for analysis of system use. It synchronises multiple video streams (left) and a visualisation of the combined log data from the PDAs and the server, via a VCR-like control panel (bottom right) or a log search tool (centre right).

with the PDA stylus, and then the players demonstrated these same manoeuvres. They were also informed that they would be observed and recorded as they played. When the participants seemed confident with the game, the players and the researchers all walked out into the game arena. Before playing each game, we let the players walk around and pick up coins in a 'pre-game'; they were able to exercise the main game operations such as uploading coins, hitting mines, pickpocketing and using the shield. Players arriving for their second or third games began out on the lawn with a reminder of the game's basics, and then they joined in the 'pre-game'. When we were sure that everyone understood the scope of the game, we played for 15 minutes. The winning team received a small prize for reasons of motivation.

\subsection{Data Collection and Analysis}

Data was collected in several ways. First of all, game related data from each game client and from the game server was logged. A tool called Replayer was developed for 
the purpose of combining the log data, and playing it back in a map-like visualisation. Replayer has a graphic interface that illustrates the progress of the game, supports queries of game state, and enables the log data to be synchronized with video streams (Fig. 2). This helped us handle the complex and often inconsistent game state data on each PDA. Potentially, each player's PDA may show a different game state at any given time, because each is based on the player's position and network connectivity, and the PDA will gain updates of game state only when inside the wireless network. Also, if a player moves outside the network, his or her GPS data cannot be sent to the server, and so the server will broadcast the player's last received position until he or she re-enters the network.

Two video streams of each game were also recorded, one from the game arena itself taken by a researcher literally running around, tracing the players and another stream taken from a window above the lawn with a good view of two thirds of the game arena. These were synchronized with the logs by Replayer, enabling analysis where both game play data and video streams were shown on one monitor simultaneously. Another researcher in the arena observed the games, and used a voice recorder to take notes about direct interaction between players. She also provided support if players had technical difficulties. After each game, the participants were interviewed in teams. This was done separately in order to get the individual teams' experiences without the other team listening in. The interviews concentrated on the enjoyment of the game and the collaboration between the teams, and on tactics and strategies that teams used. The interviews were all recorded, and transcribed shortly thereafter for analysis.

Reviewing numerous types of data such as our video recordings, system logs and post-play interviews can be challenging but, by going through each game with Replayer, it was straightforward to find features of particular interest by looking at the two video streams of the game play along with the visualised log data. On observing some feature of interaction in the video, one could check if the game state might have been an influence. Similarly, it was useful to search for certain events such as mines going off, in order to see players' reactions. We also looked for specific patterns in their collection of coins and interaction with each other. After the interviews had been transcribed, both interviews from each game were analysed by categorising topics and comparing the players' actions with game events. Overall the analysis was done on a fine-grained level. For example, the movements and the reactions of the players were described and their social interaction was tightly observed. However, because of the extensive material, we focused on issues relevant to the game experience and the issues described here such as tactics, strategies and collaboration.

\section{$5 \quad$ Results}

The games were popular and, after playing, all the participants said that it had been a fun experience. In successive games, teams became competent in the basic mechanics of game play. They became familiar with every game feature, including the more 'seamful' ones, and reported that they did not have as many 'beginners' problems' 
such as having difficulty finding a network signal or mistakenly thinking that one could pickpocket one's teammate. A number of basic statistics are shown in Fig. 3. When first-time players played against second- or third-time players, the more experienced team generally won. As they became more experienced, they also became more excited, engaged and competitive in the game, and tried hard to excel with a combination of speed and strategies. There were also a number of subtler changes in game play. As players influenced and interacted with each other, they changed how they used individual game features and how they related those features to each other.

Perhaps the most marked trend was that players chose to pickpocket much more often in later games. Second- and third-time players interacted considerably more with each other, not just through pickpocketing but also through talk, gesture and other more general forms of social interaction. We also observed that as one team-often a more experienced team-pickpocketed another heavily, the latter team would respond by using the pickpocket more. As pickpocketing increased, players used shields more and made fewer collaborative uploads. They reported that they felt more confident in their understanding of the game, and became more aware of the risk of having coins stolen. They then preferred to spread out to pick up more coins, and to upload quickly and independently to gain points. To this end, they often hit the Upload button repeatedly and were less careful to be within network; players had learnt that there was no major loss in trying to upload outside the network. Also, on average, players avoided mines better, attempted to upload more frequently and tended to play within a slightly smaller part of the arena as they became more familiar with the game.

We can focus on individual teams and players to give more detail example of how play changed over time. Team B was the only team to win three games, although they never played against a team with more experience than them. In their first game, Team B efficiently used collaborative uploads, succeeding three times, but then they began to change their play. They altered as game play evolved and opponents picked up on features such as pickpocketing. In their later games, their opponents were pickpocketing them so often that they did not upload collaboratively once. The opponents in their second game successfully pickpocketed them three times, and the opponents in their third game succeeded eleven times. Team B pickpocketed in return, succeeding eleven times in their third game also. One of the members of this team commented that their last game had been a 'button-bashing game', which was confirmed by the log data and further comments that echoed the character of this game: "[my team mate] just lost all of her [coins] [...] and I stole them from her who had taken it from [my team mate]...". The team mate interrupted: "We were standing besides each other, trying to upload together and then somebody pickpocketed me and then [my team mate] pickpocketed them back so he ended up with them all. So he uploaded them all instead of a collaborative one". 

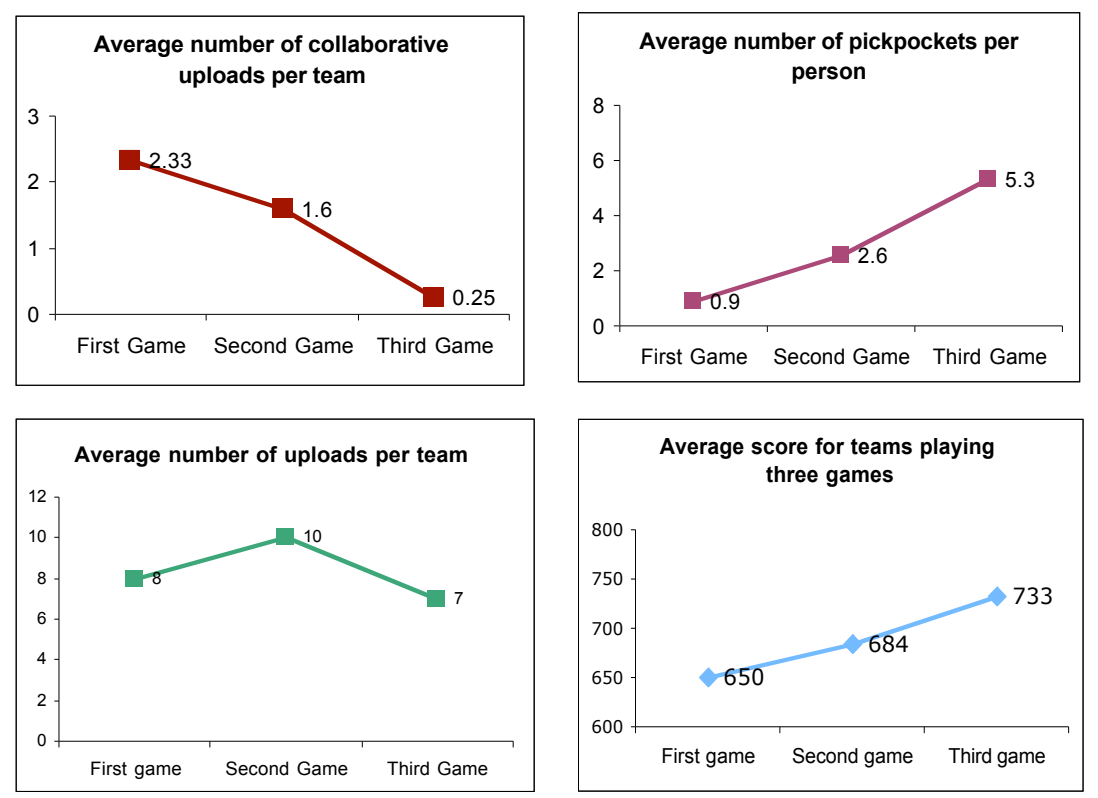

Fig. 3. Scores and trends in the games

The trial participants seemed to achieve what Salen and Zimmerman [10] call 'meaningful play' through their experience in that, through multiple plays, the relationships between actions and outcomes were both discernible and integrated into the larger context of the game. For example, one player said after the first game that she did not like the game very much, because it was difficult to find out where the signal was and where she was on the map. After the last game, however, she was asked which one of the three games she had enjoyed the most and replied: "This one [...] because you are more aware of all the things that are going on. You feel more in control of, like, what you are doing rather than just randomly pressing buttons". She and her teammate laughed and chatted as they played and won their third game. They made no collaborative uploads but did pickpocket their opponents ten times, in contrast to the two pickpockets they made in their first game.

Over the course of their games, several teams became more intense and competitive (for example, running more) whereas a few became more relaxed and cheerful. Teammates' interaction with each other appears to have been affected by the type of relationship the team members had. Although we attempted to only pair people who knew each other, two games had one team consisting of participants who did not know each other previously. In these games we observed less interaction among those team members, and only one of these games had a collaborative upload.

We now consider recurrent and significant patterns of use of game features, especially by third time players. Tactics and strategies often characterised particular subgroups of players and, we suggest, largely constituted meaningful play in the game. 


\subsection{Tactics}

Several tactical movements were observed repeatedly during the trials of the game, through direct observation and post-trial video analysis. By tactics we refer to the game-specific movements or short-term actions that players used in the game. Tactics expressed competitiveness and were often used in order to win. Most of them related to the search for coins and uploading, however others were specific to, for example, pickpocketing.

One such game-specific movement was the $180^{\circ}$ turn, which we observed three to four times per game. A player walks along, staring down at the PDA and then suddenly, without looking up, turns $180^{\circ}$ and walks in the opposite direction as if nothing had happened. When asked why they did this, we found that this was often a reaction to the player's icon passing over a coin without the player managing to pick it up. The player therefore turned around to pass over it again and pick it up.
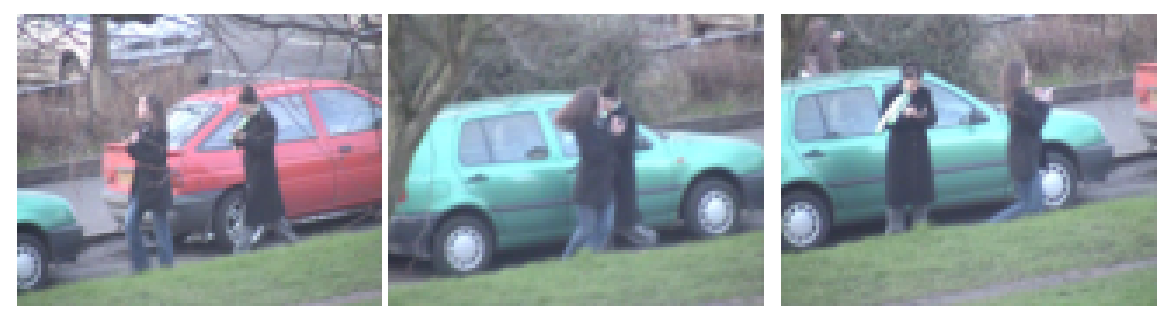

Fig. 4. A player does a $180^{\circ}$ turn while trying to find network connectivity

Because of the lag in GPS, the movement of a player's icon on the PDA was often delayed by several seconds-resulting in problems picking up coins. Most of the players learned this over the course of their games. Another reason for turning around in this way was that a player would suddenly found him- or herself leaving the network. One participant was about to upload with her teammate, but the network connection became weak; she turned 180 degrees to get back into the network (Fig. 4).

Another frequently observed move was the 'spy look'. Since players' eyes were locked to their PDAs for most of the game, and with limited visibility beyond the open lawn, players mostly judged others' position via the map on the PDA. They would stand still for a couple of seconds, look up and then around as if to see who (if anyone) was nearby, then look down and continue walking. The movement was a scanning of the environment, trying to match the information on the screen to the actual positions of the other players. Players were aware that an opposing team member could sneak up on them, without being visible on the screen, and so they would check for this. The contrary also occurred: the screen showed others close to a player and he or she then had to check if they were actually there.

A regular tactic, especially for the first-time players, was a collaborative search for network. Often team members would meet up to do a collaborative upload, but if one or both were outside the network they would walk side by side, staring down at their 
PDAs to be sure of when they had adequate connectivity to upload. Fig. 5a illustrates this tactic.

Another more energetic tactic that was observed several times in first time games consisted of players sneaking up on their opponents, pickpocketing them and then running away, so that the opponents could not steal the coins back (Fig. 5b). Although shields were widely used, the thieves either did not have them charged or thought it more efficient to run. Peculiarly enough, this seemed to be a good strategy even though the lag in GPS updating would make it inefficient. In two instances the runners succeeded and were not pickpocketed back.

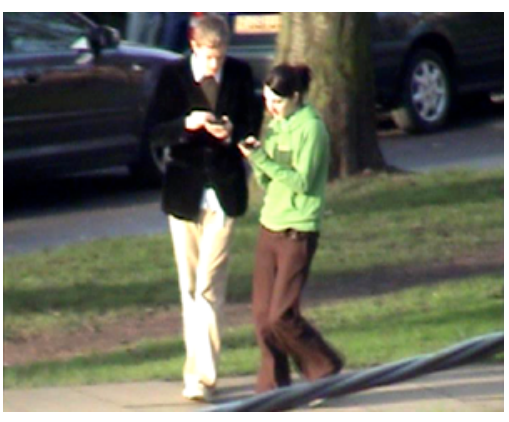

Fig. 5a. Two teammates do a collaborative search for network cover

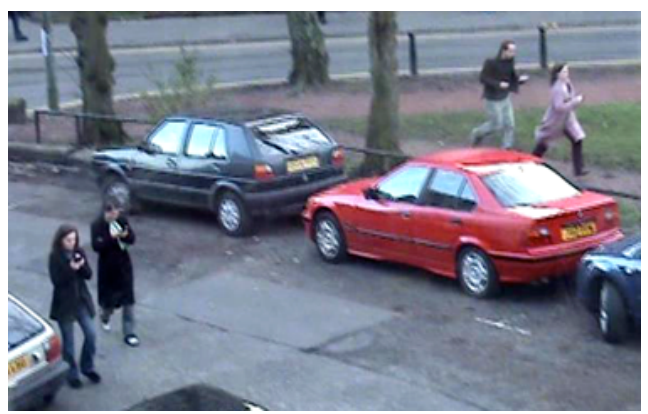

Fig. 5b. Two teammates (right) pickpocket their opponents and then run away

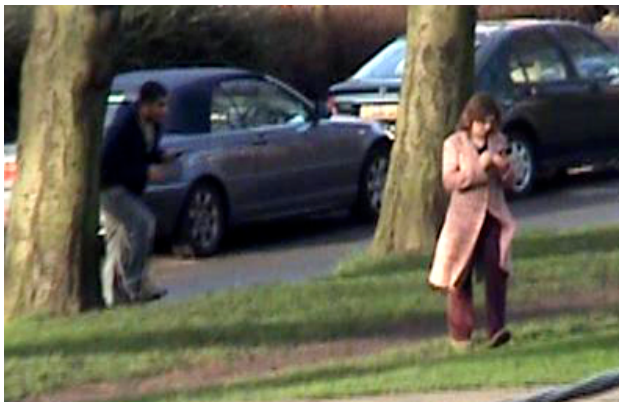

Fig. 6a. A player (left) hides behind a tree to pickpocket his opponent

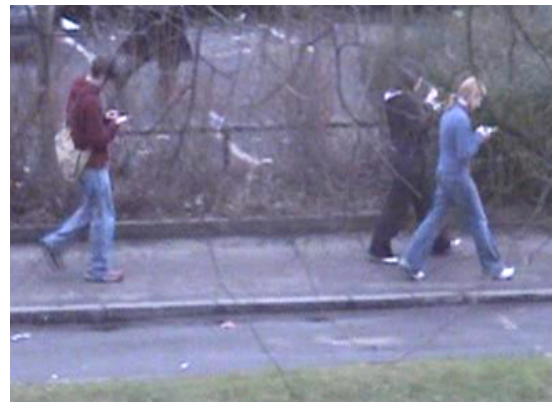

Fig. 6b. A player tracks his opponents openly, trying to pickpocket them

A complementary pair of tactics was identified through log analysis and interviews, and related to the players' level of 'sophistication' when picking up coins. Some players would carefully walk towards a coin, pressing Pickup only when their icon showed to be on top of the coin or very close to it, whereas other players would constantly and almost aggressively press the pickup button in case they were on top of a coin. Which tactic worked best is hard to say, since the points gained depended only on how many of the coins were uploaded, not how many attempts it took to pick them up. However, use of the former strategy was correlated with higher final scores. It also seemed that the players would use the aggressive technique more often when in 
a coin saturated area, whereas they would be slower and more careful when few coins were nearby. The interesting issue in this relation is that the range for picking up coins is bigger than 'being on top of it'. If the coin is within 10 meters of the player, according to GPS, it will be picked up - but GPS can jitter and lag. Combining this with the fact that new coins are being dropped constantly during the game can make the more aggressive tactic useful in some situations. The players who highlighted their use of this tactic in the interviews were all third-time players, however, not all of them were the winners of their game.

The combination of GPS inaccuracy and lag, and the game's principle of players moving outside and inside the wireless network, meant that players often experienced an inconsistency between the information on-screen and the environment. Indeed, some players commented on this point after the games, even though they had been told that such inconsistency would occur. However, most of the players quickly learned to cope with this inconsistency between what they saw on-screen and what they saw around them. As a result, some players were quick to utilise it and hide behind a tree to pickpocket innocent opponents on the lawn, or even openly follow the opponents on the path to pickpocket them (as illustrated in Fig. 6). Because some players realised that what was on their screen was not the same as what appeared on the others' screens, they played more boldly, raising their shields and attempting to steal. The games reported as most pleasurable were often the games with high rates of pickpocketing, although high levels of pickpocketing attempts were a winning tactic only if the opposing team did not also attempt to pickpocket frequently.

\subsection{Strategies}

Strategies are what we call well-considered or even planned ways of playing a game, involving longer-term activity than tactics. As they played more games, players' better understanding of the game rules, experience with the network and the familiarity with the area helped them develop strategies. We uncovered strategies through analysis of log, video and interview data as well as through observation during games. They became easier to identify as the players gained experience, because they were more distinct, the players often consciously decided upon one before the game and they could articulate them in the interviews.

The two main strategies we identified related to how players collected and uploaded coins: players were generally classified as either hunters or gatherers, and hunting seemed to work better than gathering. Hunters were the players who boldly picked up as many of the coins as they could, often from a wide area, before finding a network connection to upload. They uploaded less frequently and more carefully during the game, making sure that they were inside network coverage and had their shields on before attempting to upload-so they would not be pickpocketed. We identified six players using this strategy in one or more of their games. The trend was that the players would go 'hunting' for coins, taking chances in order to build up a number of coins for a collaborative upload with their teammate. Defined by having less than 20 attempts to upload, along with a high success rate (over 50 percent) as well as many 
coins for each upload the hunters did very well. In their second game, their average score was 455 while the average over all second games was 325 . In their third game, their average score was 480 while the average over all third games was 367 . One noteworthy thing is that the hunters did not necessarily occur in the same team: only in one team were both players identified as hunters.

Gatherers, in contrast, uploaded their coins as they picked them up, unless they had to leave the network to pick up coins - and they normally did not go far to do this. This was very much a beginner's strategy, but several players persisted with it throughout their games. Some players, shifted from gathering in the beginning of the game to hunting and collaborative uploads as play progressed and competition increased. Gatherers were very worried about getting pickpocketed, and they hit the Upload button frequently as soon as they had picked up a coin or two, attempting to upload their coins quickly. Four players were identified as using this strategy in at least one of their games. Team A serves as an example of gatherers, always trying to upload coins together and rarely going outside the network, even though many coins were located further away. This strategy initially seemed to work; they were inseparable during their third game and won by 290 points against a team of second game players. However, they did not succeed in uploading collaboratively once in that game, but instead used shields frequently and uploaded individually as soon as they had the chance. This game was dominated by an intense level of pickpocketing; team A managed to pickpocket their opponents ten times, and were pickpocketed eight times.

\section{Discussion}

The players in the trials of our game enjoyed themselves and, within the limited scope of our trials, they expressed more enjoyment in later games. This is a simple but important point to make when discussing a game, as technical novelty can wear off very quickly. Players became proficient with the basic mechanics of the game, many of which relied on seamful infrastructure features. However, their engagement with the game went beyond that: they developed tactics and strategies that built on their practice and changed their ongoing experience. They created meaningful play; the details of their play show how competitive game features were combined and traded off against collaborative features, for example. To some extent, pickpocketing was the most exciting and influential aspect of game play, and key to players' development of their two predominant strategies, hunting and gathering. The pleasure and fear of pickpocketing changed their attitude towards collaborative uploads. Even though collaborative uploads might produce very high or 'optimal' scores, pickpocketing and defending against pickpocketing were generally seen as more important.

This is just one example of the way that the experience of a game is not only about optimising progress towards a goal. It is also about weighing system features against each other, as well as shaping and reacting to the emerging behavior of opponents and teammates. Although a feature may initially appear useful in one regard, such as collaborative upload, that feature may be perceived and used differently as experience grows and social interaction continues. In many other ubicomp systems, 
this is also the case. For example, pre-existing infrastructures and the 'old media' environment have been shown to influence new infrastructures in ways that were not anticipated by designers $[1,6]$. We take a historical view of context [7], namely that past social interaction, as well as past use of the heterogeneous mix of media, tools and artifacts that make up users' activity, influence users' ongoing interaction. Patterns of use temporally and subjectively combine and interconnect different media. While we do not consider designers to be outside this process, system features used in competitive situations may influence each other in unexpected ways as players accommodate and appropriate those features, i.e. using the designers' system in their context for their uses.

This historical process was, in essence, how players came to interpret and use the new media of the game with the old media of the urban environment. More than other ubicomp systems we are aware of, the game was designed to 'seamfully' reveal 802.11 infrastructure as part of normal use. System use also revealed important features of the game arena, such as difficulty of terrain and likely visibility between players. Objectively speaking, the system affords many possible ways to play, but players' practical understanding of the system and the setting was developed through their experience of interactions with each other as much as their interactions with the system and the space. Through embodied interaction within games, as well as reflective discussion in between games, they shaped each other's interpretation of the space and the technology in a historical and intersubjective way.

Far from being a purely abstract or theoretical issue, we suggest that there are generalisable and practical issues that stem from this point in the areas of evaluation and design of ubicomp systems. One can choose to evaluate a system so as to articulate the detail and degree of change in people's use of it with ongoing activity. A substantial number of papers on ubicomp systems (including some of our own past papers) have relied on one-off plays in short demonstration games, but our experience with Treasure has reinforced our opinion that user trials of such games, and of ubicomp systems more generally, should involve repeated use and/or use over a longer time than a single, short session.

Similarly, one can choose to design a system so as to articulate the detail and degree of change in people's use of it with ongoing activity. We see the development of tactics and strategies as something that we should design for in ubicomp. We intend to build on our experience of the Replayer tool, which we found to be extremely helpful in our analysis of game play and system use, to make such replays part of the game experience in itself. Many console games, such as EA Games' Burnout for example (www.eagames.com), rely on playbacks as key elements of the user experience design. Richly instrumented code on each player's device and on the server, along with support for integrating still images and video, may then support the development of system use and also be part of system use. In other words, recording and replaying system use may aid in developing tactics and strategies for future play, but may also be an important means for players to show each other how they played in the past.

Design that makes the system so starkly open to analysis by users may seem contradictory to the design goal of 'invisibility' or 'transparency' usually associated with ubicomp, but people use past, present and potential activity involving the system in 
developing their understanding of it, i.e. as a tool they use in making the system transparent. However, Weiser's narrow design focus, concentrating only on transparent use, is at odds with the findings of user studies of how people develop their use of ubicomp systems through experience of both transparent and analytic use, and it clashes with the theory from which the transparency ideal was drawn [7]. Transparent and analytic use are mutually interdependent, with the former unavoidably influenced by analytic activity such as handling 'breakdowns', working on or adapting it, learning about it, teaching others how to use it, considering how to act so that it works better, and considering how to present oneself to others through it. Our ongoing work on making the recording and replay of system use be a resource for users' interaction is motivated by this conceptual point as much as by our experience of deployed ubicomp systems.

Lastly, we are aware of the issue of scaling of game play and system infrastructure. Our game is, at present, relatively constrained in the size of the game arena and the number of players. We have used several access points, with a virtual private network to connect them together, to create a larger game arena, and it is possible that we might be able to scale to still larger areas by using, for example, a campus-wide 802.11 network. However, our system involves a central game server and the broadcasting of all game state in regular 'heartbeat' transmissions. One possible response would be to replicate servers, and to locally partition and prioritise state broadcasts. However, we are now exploring a different direction and a different set of games that take advantage of the increasingly storage of mobile devices and their ability to set up mobile ad hoc networks. Our new games use peer-to-peer methods to disseminate and access data, and encourage more wide-ranging and opportunistic use of access points in urban areas that afford access to fixed networks as well as direct ad hoc interaction between players (and their PDAs) in the street.

\section{Conclusion}

In this paper we presented a mobile multiplayer game, Treasure, that uses spatial variation in 802.11 infrastructure as an explicit feature of its design. We set out to find how game play changed in the course of multiple games, as players' experience influenced their tactics and strategies. Mobile multi-user systems can be hard to record and evaluate in detail, and multiple plays can increase evaluation cost and workload, but we found the effort worthwhile. By looking at video of users' behaviour as well as detailed logs of system data, we obtained insights into the emergence and success of different strategies, and how features of the system and the setting were used in players' interaction with each other. As their understanding of the game grew, they used game features in different proportions, combinations and patterns. These changes in their game play did not always result in scoring more points, but they did generally lead to more excitement and engagement in the game. It is important to note that players' development of game play did not stem solely from the space the game was played in, the system design alone, or the space and the system together. Instead, players' use of this mix of old and new media developed through a historical and social 
process. Over time, people affected and were affected by each other, and system and space served as resources as well as constraints on interaction. This leads us to suggest that system designers may do more to support the development of tactics and strategies by recording data on system use and reusing it within the user experience.

Overall, we believe that our game was successful, in that variations in ubicomp infrastructure were presented and used in ways that were crucial to user engagement and enjoyment. It adds to the evidence that ubicomp infrastructure can or should be considered not only as a technical artifact but as a resource for users' interaction-interaction with the system and interaction with each other. We do not suggest that ubicomp systems should always be designed in seamful ways. Instead, we propose that new design opportunities can be found when researchers and developers consider this approach as an option. Future research may establish whether generalisation of this approach to other application areas than games is fruitful. However, our own plans for future research focus on games, as we consider them to be an area that serves well in demonstrating and generating new design ideas, engaging users in creative and intensive use of new technology, and driving the development of new infrastructure for communication, storage and computation.

\section{References}

1 Barkhuus, L., Dourish, P., Everyday Encounters with Context-Aware Computing in a Campus Environment. Proc. Ubicomp (2004) 232-249.

2 Benford, S. et al. The error of our ways: the experience of self-reported position in a location-based game. Proc. Ubicomp (2004) 70-87.

3 Benford, S. et al. Bridging the Physical and Digital in Pervasive Gaming. Communications of the ACM. Vol. 48, issue 3 (2005) 54-57.

4 Brown, B. et al. Lessons from the Lighthouse: Collaboration in a Shared Mixed Reality System. Proc ACM CHI (2003) 577-584.

5 Chalmers, M. et al., Seamful Design: Showing the Seams in Wearable Computing, Proc. IEE Eurowearable (2003) 11-17.

6 Chalmers, M. and Galani, A. Seamful Interweaving: Heterogeneity in the Theory and Design of Interactive Systems. Proc. ACM DIS (2004) 243-252.

7 Chalmers, M. A Historical View of Context. J. CSCW vol. 13 (2004) 223-247.

8 Falk J., Ljungstrand P., Björk S., Hansson R. Pirates: proximity-triggered interaction in a multi-player game. Proc. ACM CHI (2001) 119-120.

9 Flintham M. et al.. Where online meets on the streets: experiences with mobile mixed reality games. Proc. ACM CHI (2003) 569-576.

10 Mitchell M. et al. Six in the city: Introducing Real Tournament-a mobile IPv6 based context-aware multiplayer game. In Proc. ACM NETGAMES '03 (2nd workshop on network and system support for games) (2003) 91-100.

11 Salen, K., Zimmerman, E. Rules of Play: Game Design Fundamentals, MIT Press (2004)

12 Weiser, M. Building Invisible Interfaces. Keynote talk, Proc. ACM UIST (1994) 